\title{
Spin propensity in resonant photoemission of transition metal complexes
}

\author{
Robert H. Temperton $\odot,{ }^{1,2}$ Wilson Quevedo $\odot,{ }^{3}$ Robert Seidel $\odot,, 4$ Jens Uhlig $\odot,{ }^{2,5, *}$ \\ Joachim Schnadt $\odot,{ }^{1,2,6, \dagger}$ and Petter Persson ${ }^{2,7, \ddagger}$ \\ ${ }^{1}$ MAX IV Laboratory, Lund University, Box 118, 22100 Lund, Sweden \\ ${ }^{2}$ Lund Institute of Advanced Neutron and X-ray Science, IDEON Building: Delta 5, Scheelevägen 19, 22370 Lund, Sweden \\ ${ }^{3}$ Helmholtz-Zentrum Berlin für Materialien und Energie, Albert-Einstein-Straße 15, 12489 Berlin, Germany \\ ${ }^{4}$ Department of Chemistry, Humboldt-Universität zu Berlin, Brook-Taylor-Straße 2, 12489 Berlin, Germany \\ ${ }^{5}$ Division of Chemical Physics, Department of Chemistry, Lund University, Box 124, 22100 Lund, Sweden \\ ${ }^{6}$ Division of Synchrotron Radiation Research, Department of Physics, Lund University, Box 118, 22100 Lund, Sweden \\ ${ }^{7}$ Division of Theoretical Chemistry, Department of Chemistry, Lund University, Box 124, 22100 Lund, Sweden
}

(Received 8 April 2021; accepted 7 June 2021; published 7 July 2021)

\begin{abstract}
Resonant photoelectron spectroscopy (RPES) has been used to probe electronic structure properties of the closed-shell $\left[\mathrm{Fe}^{\mathrm{II}}(\mathrm{CN})_{6}\right]^{4-}$ and open-shell $\left[\mathrm{Fe}^{\mathrm{III}}(\mathrm{CN})_{6}\right]^{3-}$ prototype transition metal complexes in aqueous solution. Site-selective $\mathrm{Fe} 2 p$ ( $L$-edge) RPES maps provide new insight into spin-coupling processes at the core-excited metal centers, with autoionization of $\left[\mathrm{Fe}^{\mathrm{III}}(\mathrm{CN})_{6}\right]^{3-}$ showing a dramatic $(\sim 4 \times)$ singlet versus triplet final-state enhancement. This shows that RPES provides unique opportunities to study spin-dependent electronic properties in transition metal based functional materials.
\end{abstract}

DOI: 10.1103/PhysRevResearch.3.033030

\section{INTRODUCTION}

Resonant photoelectron spectroscopy (RPES) belongs to a class of core-hole spectroscopy methods capable of probing local electronic structure and ultrafast charge transfer dynamics $[1,2]$. As such, RPES has provided valuable contributions to our understanding of, for example, materials relevant to solar energy conversion [3-6]. It is known from early studies that resonant excitations can probe the electronic properties of different core-excited states, where the subsequent autoionization processes can manifest significant spin-selectivity [7-11]. Specifically, resonant photoemission studies of small molecules with open-shell ground states and/or degenerate frontier molecular orbitals, including ${ }^{3} \mathrm{O}_{2}$ and $\mathrm{NO}_{2}$, have been shown to probe spin-selectivity, which has been rationalized by spin propensity selection rules [12-14].

Resonant core-hole spectroscopy techniques, including $\mathrm{X}$-ray absorption spectroscopy (XAS) and resonant inelastic $\mathrm{X}$-ray scattering (RIXS), have been used to probe the local electronic structure properties of many different transition metal systems, including magnetic surfaces $[15,16]$, photocatalysts [17,18], molecular magnets [19,20], and biochemical species [21,22]. Specifically, transition metal complexes are

\footnotetext{
*jens.uhlig@chemphys.lu.se

†joachim.schnadt@sljus.lu.se

†petter.persson@teokem.lu.se
}

Published by the American Physical Society under the terms of the Creative Commons Attribution 4.0 International license. Further distribution of this work must maintain attribution to the author(s) and the published article's title, journal citation, and DOI. widely used in photochemical applications [23] and as molecular magnets [24] due to their ability to sustain a variety of spin and oxidation states [25]. Valuable information about both the metal and ligand electronic structure properties of various transition metal complexes have been studied by RIXS [26-28] and RPES [29,30].

As a further step toward a comprehensive understanding of electronic and spin properties of transition metal complexes, we here present high-resolution RPES maps probing the central iron atom in the prototype transition metal complexes $\left[\mathrm{Fe}^{\mathrm{II}}(\mathrm{CN})_{6}\right]^{4-}$ and $\left[\mathrm{Fe}^{\mathrm{III}}(\mathrm{CN})_{6}\right]^{3-}$. This enables a detailed electronic structure comparison between the $\mathrm{Fe}^{\mathrm{II}}$ closed-shell and $\mathrm{Fe}^{\mathrm{III}}$ open-shell forms of otherwise chemically identical molecules. We show that RPES mapping of the metal site provides a detailed probe of the local electronic structure and, in particular, it provides unique insight into the spin-selectivity of different oxidation states encountered in the resonant decay processes. This is not accessible by other resonant core-hole spectroscopy techniques such as XAS or RIXS. This paper evaluates spin propensity as a continuous function of photon energy, enabling a detailed experimental exploration of the coupling between electronic arrangements in the intermediate and final states.

Both $\left[\mathrm{Fe}^{\mathrm{II}}(\mathrm{CN})_{6}\right]^{4-}$ and $\left[\mathrm{Fe}^{\mathrm{III}}(\mathrm{CN})_{6}\right]^{3-}$ complexes have an octahedral geometry where the central $\mathrm{Fe}$ atom is influenced by a strong and highly symmetric ligand field. In accordance with ligand field theory, the partially occupied $3 d$ orbitals split in energy, producing two well-separated sets of orbitals, designated as $t_{2 g}$ and $e_{g}$ [31] (see Fig. 1). The strength of the $t_{2 g} / e_{g}$ energy splitting means it is energetically favorable for the molecules to be in a low-spin configuration with doubly occupied $t_{2 g}$ and unoccupied $e_{g}$ orbitals. In the $\mathrm{Fe}^{\mathrm{II}}$ 


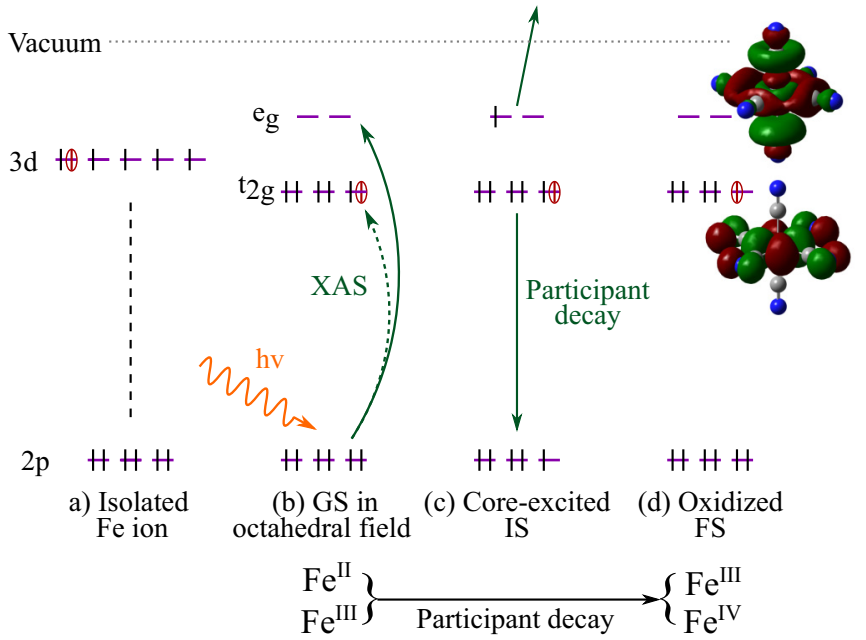

FIG. 1. Illustration of the electronic structure, resonant excitation, and participant decay of the Fe complexes. The red marking indicates an electron for the closed-shell $\left[\mathrm{Fe}^{\mathrm{II}}(\mathrm{CN})_{6}\right]^{4-}$ and a hole for the open-shell $\left[\mathrm{Fe}^{\mathrm{III}}(\mathrm{CN})_{6}\right]^{3-}$. (a) An isolated $\mathrm{Fe}$ ion with degenerate $3 d$ orbitals. (b) The ground state $(\mathrm{GS})$ of $\left[\mathrm{Fe}^{\mathrm{II} / \mathrm{III}}(\mathrm{CN})_{6}\right]^{4-/ 3-}$ where the octahedral ligand-field splits the $3 d$ into $t_{2 g}$ and $e_{g}$ orbitals. (c) The core-excited intermediate state (IS) after an Fe $2 p \rightarrow e_{g}$ photoexcitation with the resulting participant decay indicated. (d) The oxidized final state (FS) with an additional hole in the $t_{2 g}$, where representative plots of the $t_{2 g}$ and $e_{g}$ are inset.

ground state, the $\mathrm{Fe}$ atom is therefore in the valence singlet configuration ${ }^{1}\left|\left(t_{2 g}\right)^{6}\left(e_{g}\right)^{0}\right\rangle$. The $\mathrm{Fe}^{\mathrm{III}}$ ground state, however, has a partially unoccupied $t_{2 g}$, described by the ${ }^{2}\left|\left(t_{2 g}\right)^{5}\left(e_{g}\right)^{0}\right\rangle$ valence doublet state. This option to have a hole in the $t_{2 g}$ is indicated in Fig. 1.

Following photoexcitation, in which the absorption of an $\mathrm{X}$-ray photon leads to the promotion of an $\mathrm{Fe} 2 p$ electron to the first unoccupied $\mathrm{Fe} 3 d$ orbital $\left(e_{g}\right.$ for $\mathrm{Fe}^{\mathrm{II}}$ or $t_{2 g}$ for $\mathrm{Fe}^{\mathrm{III}}$ ), one of the possible relaxation processes is participant decay [1]. Here, the photoexcited electron and a second valence electron participate in an autoionization decay process in which one of the electrons is emitted and the other repopulates the $\mathrm{Fe} 2 p$ core hole. The kinetic energy of the emitted electron can be measured, and the molecule is left in an oxidized final state. Molecular orbital plots showing the character of the $t_{2 g}$ and $e_{g}$ orbitals in this final state are inset into Fig. 1.

Given that the participant decay channel is only possible when the incident photon precisely matches an electronic transition, the participant intensity is therefore an indication of the coupling strength between a particular intermediate core-excited state and the resulting final state. RPES allows the exploration of this energy landscape where the intensity at any point is governed by the Kramers-Heisenberg equation [32]. The two main classes of spectral features in RPES result from direct photoemission (through the photoelectric effect) and autoionization, of which participant decay is a specific example.

This article applies the principle of spin propensity in the final state of RPES, pioneered for the case of small gasphase molecules [12-14], to a transition metal complex with a significantly more complicated electronic structure. In this work, we demonstrate how the application of spin propen- sity selection rules in RPES can help to explain the spectral features of open-shell transition metal complexes more generally, and we highlight how two-dimensional RPES mapping can be used to uncover otherwise experimentally inaccessible information.

\section{RESULTS AND DISCUSSION}

Liquid-jet RPES measurements of aqueous solutions of $\mathrm{K}_{3} \mathrm{Fe}(\mathrm{CN})_{6}$ and $\mathrm{K}_{4} \mathrm{Fe}(\mathrm{CN})_{6}$ were conducted using the SOL ${ }^{3}$ PES experimental station [35] and the U49/2-PGM-1 beamline [36] at the BESSY II synchrotron radiation facility. Further experimental details can be found in Appendix A. The Fe $2 p$ L-edge spectra are presented in Fig. 2, where the top and bottom series of plots show measurements of $\left[\mathrm{Fe}^{\mathrm{II}}(\mathrm{CN})_{6}\right]^{4-}$ and $\left[\mathrm{Fe}^{\mathrm{III}}(\mathrm{CN})_{6}\right]^{3-}$, respectively. The unprocessed RPES maps, showing the valence-band PES measured as a function of incident photon energy, are shown on the left in panels (a) and (b). High-resolution spectra, shown in (c) and (d), were measured both off and on key resonances visible in the x-ray absorption spectra, shown in (e) and (f). The plots at the right of the panel, $(\mathrm{g})$ and $(\mathrm{h})$, show RPES difference maps created by subtracting the pre-edge valence-band spectrum from the raw data in (a) and (b). This separates the Fe resonant enhancements from the direct photoemission signal, which is dominated by photoemission from the water solvent.

For $\left[\mathrm{Fe}^{\mathrm{II}}(\mathrm{CN})_{6}\right]^{4-}$, the lower-energy peak in the XAS at $\sim 711 \mathrm{eV}$ is dominated by $2 p \rightarrow e_{g}$ transitions, both with and without $t_{2 g} \rightarrow e_{g}$ shakeups. The higher peak at $\sim 713 \mathrm{eV}$ originates from a mix of transitions into metal and ligand states, which include $\pi$ back-bonding and spin-forbidden singlet-triplet transitions that have a notable intensity due to spin-orbit coupling $[27,37,38]$. For $\left[\mathrm{Fe}^{\mathrm{III}}(\mathrm{CN})_{6}\right]^{3-}$, the spectra are more complicated due to the open-shell nature of the molecule. The first resonance at $h v=707.5 \mathrm{eV}$ corresponds to excitation into the $t_{2 g}$ hole, while the structure in the XAS above $\sim 710 \mathrm{eV}$ is from $e_{g}$-related excitations. The shape has been explained through a combination of multiplet effects, $\pi$ back-bonding, and $\sigma$ donation. The lowest-energy transition in this complex series of $e_{g}$-derived transitions is the $2 p \rightarrow e_{g}$ resonance [27,37,39].

The nonresonant valence-band features of $\left[\mathrm{Fe}^{\mathrm{II}}(\mathrm{CN})_{6}\right]^{4-}$ and $\left[\mathrm{Fe}^{\mathrm{III}}(\mathrm{CN})_{6}\right]^{3-}$ have previously been described from a molecular orbital perspective [40]. For RPES, however, it is helpful to extend this picture by considering each enhancement, at a particular location in the two-dimensional map, as a unique coupling of a core-exited state and a final state. Very broadly, the RPES data can be separated into two binding energy regions. At low binding energy $(\lesssim 10 \mathrm{eV})$, the spectra are dominated by participant enhancement of the $t_{2 g}$ highest occupied molecular orbital (HOMO) (labeled in Fig. 2). At higher binding energies, in addition to participant enhancement of valence-band features, we also observe "spectator"/resonant Auger enhancements [41]. The "spectator" Auger, where the photoexcited electron remains in the unoccupied orbital and does not participate in the Auger decay process, will follow a constant kinetic energy trajectory $\left(45^{\circ}\right.$ angle in the maps), increasing in intensity when on a resonant transition. This paper, however, focuses entirely on the low binding energy participant enhancements. 

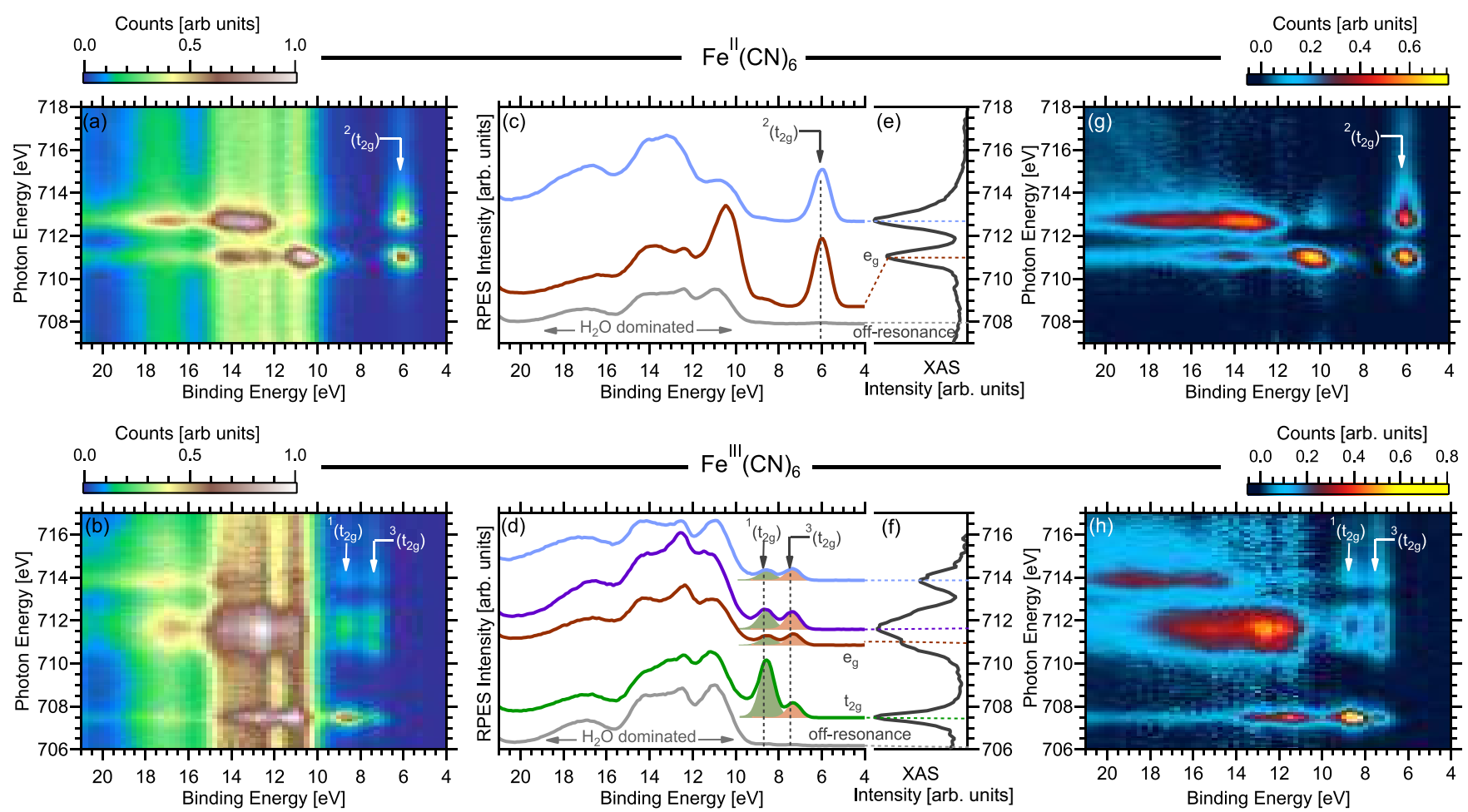

FIG. 2. Fe $2 p$ L-edge RPES of $\left[\mathrm{Fe}^{\mathrm{II}}(\mathrm{CN})_{6}\right]^{4-}$ (top) and $\left[\mathrm{Fe}^{\mathrm{III}}(\mathrm{CN})_{6}\right]^{3-}$ (bottom). (a),(b) RPES maps (raw data). (c),(d) Spectra measured off-resonance and on selected resonances in the partial electron yield x-ray absorption spectrum, shown in (e),(f) (a vertical integration over the RPES map). The $t_{2 g}$ and $e_{g}$ resonances are marked. (g),(h) Difference maps showing the RPES signal after subtracting the off-resonance valence-band spectrum [gray spectrum in (c) and (d)], which is largely dominated by the valence band of water. Binding energies were calibrated using the nearby water $2 a_{1}$ peak at $30.9 \mathrm{eV} \mathrm{[33]} \mathrm{(see} \mathrm{the} \mathrm{spectra} \mathrm{in} \mathrm{the} \mathrm{supplemental} \mathrm{material} \mathrm{[34]).}$

For $\left[\mathrm{Fe}^{\mathrm{II}}(\mathrm{CN})_{6}\right]^{4-}$ the $t_{2 g}$ participant enhancement is observed at $\sim 6.0 \mathrm{eV}$ binding energy, appearing strongly on both resonances. These enhancements correspond to the valence doublet final state ${ }^{2}\left(t_{2 g}\right)^{5}$. For $\left[\mathrm{Fe}^{\mathrm{III}}(\mathrm{CN})_{6}\right]^{3-}$, there are two notable binding energy features at 8.6 and $7.3 \mathrm{eV}$. These are assigned to participant enhancements of the ${ }^{1}\left(t_{2 g}\right)^{4}$ and ${ }^{3}\left(t_{2 g}\right)^{4}$ (singlet and triplet) final states, respectively. Interestingly, despite being enhancements of the same molecular orbital feature, their relative intensities depend on the intermediate core-excited state. Figure 3 shows vertical line

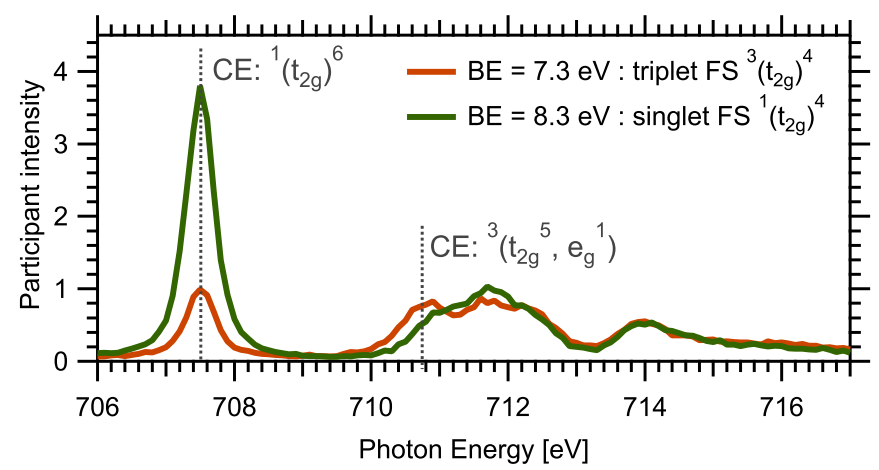

FIG. 3. Evolution of the participant enhancement of the ${ }^{1} t_{2 g}$ and ${ }^{3} t_{2 g}$ final states (FS) as a function of photon energy. These final state spectra are vertical constant binding energy (BE) line profiles extracted from the $\left[\mathrm{Fe}^{\mathrm{III}}(\mathrm{CN})_{6}\right]^{3-}$ RPES map [Fig. 2(b)]. CE denotes core-excited intermediate state. profiles extracted from the RPES map (0.5-eV-wide integrations, centered on the two features), which highlight the intensity evolution of the singlet and triplet state as a function of photon energy. On the $t_{2 g}$ resonance $(h v=707.5 \mathrm{eV})$, the valence singlet dominates. On the rising edge of the $e_{g}$ XAS feature (up to $h v \sim 711 \mathrm{eV}$ ) the valence triplet is more intense, but at its peak $(h v=712.6 \mathrm{eV})$ the singlet appears slightly more intense. At higher photon energies, both are of similar intensity. Quantification of the relative intensity of the singlet compared to the triplet for $\left[\mathrm{Fe}^{\mathrm{III}}(\mathrm{CN})_{6}\right]^{3-}$, as derived from fitting Voigt functions to the spectra in Fig. 2(d), can be found in Table I.

This complex picture of multiple core-excited (intermediate) states and final states following participant enhancement of the $t_{2 g}$ is depicted in Fig. 4. In the closed shell $\mathrm{Fe}^{\mathrm{II}}$ case [Fig. 4(a)], the $2 p \rightarrow e_{g}$ excitation can only produce the single valence doublet intermediate state ${ }^{2}\left|\left(t_{2 g}\right)^{6}\left(e_{g}\right)^{1}\right\rangle$.

TABLE I. Ratio of valence singlet/triplet participant intensity in $\left[\mathrm{Fe}^{\mathrm{III}}(\mathrm{CN})_{6}\right]^{3-}$, from peak fitting the spectra in Fig. 2(d).

\begin{tabular}{lc}
\hline \hline Photon Energy $[\mathrm{eV}]$ & ${ }^{1}\left(t_{2 g}\right) /{ }^{3}\left(t_{2 g}\right)$ \\
\hline 706.0 (pre-edge) & $1.36 \pm 0.78$ \\
$707.5\left(t_{2 g}\right)$ & $3.93 \pm 0.13$ \\
$711.0\left(e_{g}\right)$ & $0.82 \pm 0.07$ \\
711.6 & $1.17 \pm 0.08$ \\
713.8 & $0.94 \pm 0.10$ \\
\hline
\end{tabular}



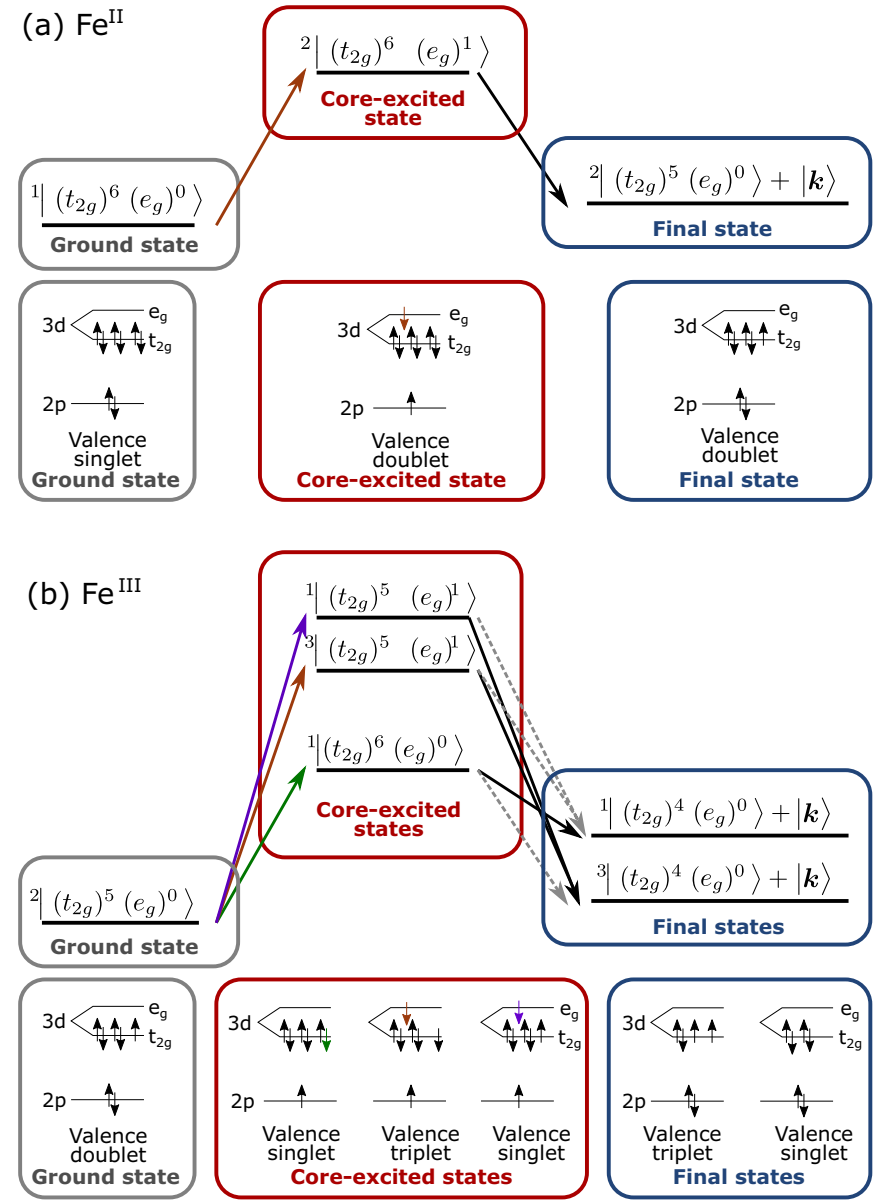

FIG. 4. Generalized state diagram depicting the relative energies and valence configurations in Fe $2 p$ RPES of (a) closed-shell $\mathrm{Fe}^{\mathrm{II}}$ and (b) open-shell $\mathrm{Fe}^{\mathrm{III}}$ low-spin complexes. The ground state, core-excited intermediate states, and final states following participant decay are shown. $\mathbf{k}$ denotes the wave vector of the emitted photoelectron. Gray dashed arrows indicate final states with diminished intensity.

Following participant decay, the $2 p$ core-hole is repopulated and a $t_{2 g}$ electron is emitted producing the valence doublet ${ }^{2}\left|\left(t_{2 g}\right)^{5}\left(e_{g}\right)^{0}\right\rangle$ final state.

The open-shell nature of $\left[\mathrm{Fe}^{\mathrm{III}}(\mathrm{CN})_{6}\right]^{3-}$ allows the electrons to be arranged with several spin-multiplicities, in both intermediate and final states, as is depicted in Fig. 4(b). First, the $2 p \rightarrow t_{2 g}$ excitation can create the valence singlet intermediate state ${ }^{1}\left|\left(t_{2 g}\right)^{6}\left(e_{g}\right)^{0}\right\rangle$. Second, the $2 p \rightarrow e_{g}$ excitation can either create a valence singlet, ${ }^{1}\left|\left(t_{2 g}\right)^{5}\left(e_{g}\right)^{1}\right\rangle$, or triplet, ${ }^{3}\left|\left(t_{2 g}\right)^{5}\left(e_{g}\right)^{1}\right\rangle$, depending on the spin of the electron in the $e_{g}$ level. After participant decay, two distinct final states are possible depending on the arrangement of the remaining $t_{2 g}$ electrons: the valence triplet ${ }^{3}\left|\left(t_{2 g}\right)^{4}\left(e_{g}\right)^{0}\right\rangle$ or the higherenergy singlet ${ }^{1}\left|\left(t_{2 g}\right)^{4}\left(e_{g}\right)^{0}\right\rangle$. The latter singlet state has two configuration options whereby two out of three $t_{2 g}$ orbitals are fully populated (as shown in the diagram) or, alternatively, one orbital is fully populated and two are half-populated. Density functional theory calculations of $\left[\mathrm{Fe}^{\mathrm{III}}(\mathrm{CN})_{6}\right]^{3-}$ predict a $1.66 \mathrm{eV}$ energy splitting between the valence triplet and the higher-energy singlet (with two doubly occupied $t_{2 g}$ levels) final states. This calculation, further detailed in Appendix B, provides a reasonable match with the $1.3 \mathrm{eV}$ splitting of the $t_{2 g}$ participant features experimentally observed in the RPES measurements.

The relative intensity of the singlet and triplet features in the RPES can be explained through a spin-propensity rule derived from the principle that transitions involving spinup/spin-down electron pairs are favorable over those with aligned spins. Similar arguments have previously been discussed in the context of small gas-phase molecules [12-14]. Applying this selection rule to $\left[\mathrm{Fe}^{\mathrm{III}}(\mathrm{CN})_{6}\right]^{3-}$, following the core-excitation into the $t_{2 g}$ orbital, it is favorable to end in the higher-energy singlet final state. This is reflected in the intensity of the participant signal presented here, which for the $\left[\mathrm{Fe}^{\mathrm{III}}(\mathrm{CN})_{6}\right]^{3-} t_{2 g}$ excitation shows that the valence singlet final state has a propensity of $3.9 \pm 0.1$ greater than that of the triplet.

On the $e_{g}$ resonances, neither final state is diminished by this propensity rule. On the rising edge of the $e_{g}$ resonance, the RPES shows higher triplet participant intensity compared to the singlet, indicating the lower energy triplet state is favorable. At further increasing photon energies, the singlet and triplet have comparable intensity. We propose that on the leading edge of the $e_{g}$ resonance, there is only enough energy to access the valence triplet core-excited state, whereas at higher photon energies the higher-energy singlet core-excited state can be accessed. It follows that the observed intensity variation of the two final states is likely a manifestation of the coupling strength between the intermediate and final states, such that the coupling to the singlet final state is stronger for the intermediate singlet state than the intermediate triplet state. This highlights the powerful capability of RPES for experimentally deconstructing complex $L$-edge XAS.

\section{CONCLUSIONS}

The results presented here highlight clear differences between the aqueous solutions of $\left[\mathrm{Fe}^{\mathrm{II}}(\mathrm{CN})_{6}\right]^{4-}$ and $\left[\mathrm{Fe}^{\mathrm{III}}(\mathrm{CN})_{6}\right]^{3-}$, beyond what can be extracted from standard x-ray absorption or photoelectron spectroscopy. The $\mathrm{Fe}^{\mathrm{II}}$ and Fe ${ }^{\text {III }}$ RPES manifests clear differences in both the occupied and unoccupied electronic structure. We show that RPES mapping can provide detailed information about the coupling of different spin configurations in the intermediate and final states. Spin propensity in the open-shell $\left[\mathrm{Fe}^{\mathrm{III}}(\mathrm{CN})_{6}\right]^{3-}$ complex is observed. Specifically, the participant enhancement of the HOMO singlet state appears $\sim 4 \times$ that of the lower energy triplet when on resonance with the $\mathrm{Fe} 2 p \rightarrow t_{2 g}$ excitation.

When compared to RIXS, the photon-in/photon-out ana$\log$ of RPES (photon-in/electron-out), the resulting final states are different. In RIXS, no electron is emitted (the final state is not oxidized) impacting the accessible spin multiplicities. While both techniques are powerful and provide complementary information, the ability of RPES to probe this different final state has remained underutilized until now. The experimental observations of orbital degeneracy and spinpropensity presented in this paper set out a platform for using RPES mapping to resolve otherwise inaccessible spin configurations. This has particular significance to understanding the 
fundamental electronic and spin properties of open-shell or high-spin materials.

\section{ACKNOWLEDGMENTS}

We acknowledge the Helmholtz-Zentrum-Berlin (HZB) and the staff at BESSY II for generously supporting our measurements. We gratefully acknowledge financial support from Vetenskapsrädet (VR, the Swedish Research Council), Energimyndigheten (the Swedish Energy Agency), the Knut and Alice Wallenbergs Stiftelse (KAW, the Knut and Alice Wallenberg Foundation), the Stiftelsen för Strategisk Forskning (SSF, Swedish Foundation for Strategic Research), as well as generous provision of computing facilities at the NSC and LUNARC computing centers via a SNIC allocation. R.H.T. is grateful for postdoc funding from Lund University. R.S. and W.Q. acknowledge funding from the Deutsche Forschungsgemeinschaft (DFG, German Research Foundation) through an Emmy-Noether Grant No. (SE 2253/3-1).

\section{APPENDIX A: EXPERIMENTAL DETAILS}

Liquid-jet photoelectron spectroscopy measurements were carried out with the SOL ${ }^{3}$ PES ambient pressure $x$-ray photoelectron spectroscopy (AP-XPS) experimental station [35] at the U49/2-PGM-1 beamline [36] at the BESSY II synchrotron radiation facility. The U49/2-PGM-1 beamline provides photons in the range of $85-1600 \mathrm{eV}$ producing a $20 \times 80 \mu \mathrm{m}^{2}$ spot of linearly polarized light at the measurement position at $\mathrm{SOL}^{3} \mathrm{PES}$. This experimental station is equipped with a Scienta HIPP-2 R4000 hemispherical analyzer, which was positioned at the magic angle, i.e. $54.7^{\circ}$ relative to the polarization axis of the incident x-ray beam. All measurements were taken in swept mode with a pass energy of $200 \mathrm{eV}$ with a theoretical binding energy resolution of $450 \mathrm{meV}$. The energy resolution of the beamline at the Fe L-edge was better than $280 \mathrm{meV}$.

$\mathrm{K}_{3} \mathrm{Fe}(\mathrm{CN})_{6}$ and $\mathrm{K}_{4} \mathrm{Fe}(\mathrm{CN})_{6}$ (from Sigma Aldrich) were dissolved in deionized water to concentrations of $0.3 \mathrm{M}$ and $0.4 \mathrm{M}$, respectively. The solutions were degassed before use. They were pumped from a reservoir by an HPLC pump (Techlab Model Economy) and injected into the vacuum chamber through a fused silica capillary of $30 \mu \mathrm{m}$ inner diameter. The laminar liquid microjet had a velocity of approximately $40 \mathrm{~ms}^{-1}$. We cooled the jet assembly down to $10^{\circ} \mathrm{C}$ using a chiller (Julabo Model F12/ED) to reduce the water vapor pressure and therefore the gas-phase spectral contributions. Approximately $1 \mathrm{~mm}$ downstream, the liquid microjet was hit by the synchrotron beam and the ejected photoelectrons passed through a $0.5 \mathrm{~mm}$ orifice that forms the entrance to the hemispherical electron analyzer at a distance of $0.5 \mathrm{~mm}$ from the liquid microjet. This short distance ensures that detected electrons did not suffer from inelastic scattering with gas-phase water molecules surrounding the jet surface. The pressure in the vacuum chamber during the measurements was better than $2 \times 10^{-4}$ mbar, which was achieved by a combination of cold traps - metal cylinders filled with liquid nitrogen - and a $1500 \mathrm{Ls}^{-1}$ turbomolecular pump.

The binding energy of the valence-band resonant photoemission was calibrated by setting the nearby water $2 a_{1}$ peak to $30.9 \mathrm{eV}$ [33] (see the spectrum in the supplemental material [34]), which is unaffected by the spectral enhancements.
Peak fitting of the valence singlet and triplet states (following participant decay) used Voigt functions where the Lorentzian width was fixed to $0.05 \mathrm{eV}$ and the Gaussian width was allowed to vary in the fit. The shape of the valence band was taken into consideration in the fit using an arbitrary series of Voigt functions with no constraints - these components are not used in any analysis, but they were included to allow the background below the singlet/triplet peaks to be accurately modeled in the fit. A Shirley background was used. An example of this peak-fitting procedure is included in the supplemental material [34].

The XAS presented in the manuscript are partial electron yield spectra extracted by vertically integrating over the RPES maps. This was done over the full binding energy range measured, from 2 to $36 \mathrm{eV}$. These raw datasets are included in the supplemental material [34].

\section{APPENDIX B: COMPUTATIONAL DETAILS}

The GAUSSIAN 09 software package [42] was used to perform density functional theory (DFT) calculations of $\left[\mathrm{Fe}^{\mathrm{III}}(\mathrm{CN})_{6}\right]^{3-}$. The B3LYP* hybrid functional [43] [generalized gradient approximation with $15 \%$ Hartree-Fock (HF) $/ 13 \%$ Slater $/ 72 \%$ Becke- 88 exchange functional and $19 \%$ VWN3/81\% LYPC correlation functional] and the def2tzvpd basis set [44] (from Basis Set Exchange [45]) were used. The B3LYP* functional is a modification of the widely established B3LYP functional with a reduced amount of HF exchange which has been shown to be well suited for complexes of first-row transition metals $[43,46]$. The water solvent was modeled using a polarizable continuum model [47]. The geometry was optimized in the ground state ${ }^{2}\left[\mathrm{Fe}^{\mathrm{III}}(\mathrm{CN})_{6}\right]^{3-}$. Single point energy calculations were conducted using this optimized geometry for the triplet ${ }^{3}\left[\mathrm{Fe}^{\mathrm{III}}(\mathrm{CN})_{6}\right]^{2-}$ and singlet ${ }^{1}\left[\mathrm{Fe}^{\mathrm{III}}(\mathrm{CN})_{6}\right]^{2-}$ final states. Both singlet final states were calculated: one with two fully occupied $t_{2 g}$ orbitals and the other with one fully occupied and two partially occupied $t_{2 g}$ orbitals. The difference in the self-consistent-field (SCF) energy between the doublet ground state and the various oxidized final-state configurations was used to determine the final-state energies. The energies of these states are shown in Table II.

TABLE II. Table of energies from DFT calculations of the initial ground states (GS) and final states $(\mathrm{FS})$ of $\left[\mathrm{Fe}^{\mathrm{III}}(\mathrm{CN})_{6}\right]^{3-} \cdot E_{\mathrm{SCF}}$ denotes the self-consistent-field energy from the calculation. $E_{\mathrm{FS}}$ is the final-state energy (the difference in SCF energy of the initial and final states). ${ }^{1} \mathrm{FS}-{ }^{3} \mathrm{FS}$ is the energy difference between the singlet and triplet final states.

\begin{tabular}{|c|c|c|c|c|}
\hline State & $\begin{array}{c}E_{\mathrm{SCF}} \\
\text { (Hartree) }\end{array}$ & $\begin{array}{l}E_{\mathrm{SCF}} \\
(\mathrm{eV})\end{array}$ & $\begin{array}{l}E_{\mathrm{FS}} \\
(\mathrm{eV})\end{array}$ & $\begin{array}{c}{ }^{1} \mathrm{FS}-{ }^{3} \mathrm{FS} \\
(\mathrm{eV})\end{array}$ \\
\hline $\begin{array}{l}\mathrm{GS} \\
{ }^{2}\left[\mathrm{Fe}^{\mathrm{III}}(\mathrm{CN})_{6}\right]^{3-}\end{array}$ & -1821.0711 & -49553.88 & & \\
\hline $\begin{array}{l}{ }^{3} \mathrm{FS} \\
{ }^{3}\left[\mathrm{Fe}^{\mathrm{IV}}(\mathrm{CN})_{6}\right]^{2-}\end{array}$ & -1820.8661 & -49548.31 & -5.57 & \\
\hline $\begin{array}{l}{ }^{1} \mathrm{FS}\left(t_{2 g}^{2}, t_{2 g}^{1}, t_{2 g}^{1}\right) \\
{ }^{1}\left[\mathrm{Fe}^{\mathrm{IV}}(\mathrm{CN})_{6}\right]^{2-}\end{array}$ & -1820.8465 & -49547.78 & -6.10 & -0.53 \\
\hline $\begin{array}{l}{ }^{1} \mathrm{FS}\left(t_{2 g}^{2}, t_{2 g}^{2}, t_{2 g}^{0}\right) \\
{ }^{1}\left[\mathrm{Fe}^{\mathrm{IV}}(\mathrm{CN})_{6}\right]^{2-}\end{array}$ & -1820.8053 & -49546.65 & -7.23 & -1.66 \\
\hline
\end{tabular}


[1] P. A. Brühwiler, O. Karis, and N. Mårtensson, Chargetransfer dynamics studied using resonant core spectroscopies, Rev. Mod. Phys. 74, 703 (2002).

[2] L. Wang, W. Chen, and A. T. S. Wee, Charge transfer across the molecule/metal interface using the core hole clock technique, Surf. Sci. Rep. 63, 465 (2008).

[3] J. Schnadt, P. A. Brühwiler, L. Patthey, J. N. O'Shea, S. Södergren, M. Odelius, R. Ahuja, O. Karis, M. Bässler, P. Persson, H. Siegbahn, S. Lunell, and N. Mårtensson, Experimental evidence for sub-3-fs charge transfer from an aromatic adsorbate to a semiconductor, Nature (London) 418, 620 (2002).

[4] C. Parks Cheney, P. Vilmercati, E. W. Martin, M. Chiodi, L. Gavioli, M. Regmi, G. Eres, T. A. Callcott, H. H. Weitering, and N. Mannella, Origins of Electronic Band Gap Reduction in $\mathrm{Cr} / \mathrm{N}$ Codoped $\mathrm{TiO}_{2}$, Phys. Rev. Lett. 112, 036404 (2014).

[5] L. C. Mayor, J. Ben Taylor, G. Magnano, A. Rienzo, C. J. Satterley, J. N. O'Shea, and J. Schnadt, Photoemission, resonant photoemission, and $\mathrm{x}$-ray absorption of a $\mathrm{Ru}(\mathrm{II})$ complex adsorbed on rutile $\mathrm{TiO}_{2}(110)$ prepared by in situ electrospray deposition, J. Chem. Phys. 129, 114701 (2008).

[6] E. M. Johansson, R. Lindblad, H. Siegbahn, and A. Hagfeldt, Atomic and electronic structures of interfaces in dye-sensitized, nanostructured solar cells, ChemPhysChem 15, 1006 (2014).

[7] J. Schirmer, L. S. Cederbaum, and W. Von Niessen, Twohole-one-particle configuration interaction approach for the ionization of open-shell molecules: Application to $\mathrm{NO}_{2}$, Chem. Phys. 56, 285 (1981).

[8] H. Ågren, On the interpretation of molecular valence Auger spectra, J. Chem. Phys. 75, 1267 (1981).

[9] H. Siegbahn, L. Asplund, and P. Kelfve, The Auger electron spectrum of water vapour, Chem. Phys. Lett. 35, 330 (1975).

[10] H. Ågren and O. Vahtras, Spin-orbit coupling in molecular auger spectra: Propensity rules tested for water, J. Phys. B 26, 913 (1993).

[11] N. Kosugi, Exchange interaction in core excitation of diatomic systems, Chem. Phys. 289, 117 (2003).

[12] M. N. Piancastelli, V. Carravetta, I. Hjelte, A. De Fanis, K. Okada, N. Saito, M. Kitajima, H. Tanaka, and K. Ueda, Experimental and theoretical study of resonant Auger decay of core-excited $\mathrm{NO}_{2}$, Chem. Phys. Lett. 399, 426 (2004).

[13] R. F. Fink, F. Burmeister, R. Feifel, M. Bässler, O. Björneholm, L. Karlsson, C. Miron, M.-N. Piancastelli, S. L. Sorensen, H. Wang, K. Wiesner, and S. Svensson, Spin-orbit selectivity observed for the $\mathrm{HCl}^{+}\left(\sim X^{2} \Pi\right)$ state using resonant photoemission, Phys. Rev. A 65, 034705 (2002).

[14] T. X. Carroll and T. D. Thomas, Deexcitation electron spectroscopy of core-excited $\mathrm{O}_{2}$, J. Chem. Phys. 92, 7171 (1990).

[15] M. Kobayashi, H. Niwa, Y. Takeda, A. Fujimori, Y. Senba, H. Ohashi, A. Tanaka, S. Ohya, P. N. Hai, M. Tanaka, Y. Harada, and M. Oshima, Electronic Excitations of a Magnetic Impurity State in the Diluted Magnetic Semiconductor (Ga,Mn)As, Phys. Rev. Lett. 112, 107203 (2014).

[16] Y. Lu, D. Betto, K. Fürsich, H. Suzuki, H.-H. Kim, G. Cristiani, G. Logvenov, N. B. Brookes, E. Benckiser, M. W. Haverkort, G. Khaliullin, M. Le Tacon, M. Minola, and B. Keimer, Site-Selective Probe of Magnetic Excitations in Rare-Earth Nickelates Using Resonant Inelastic X-Ray Scattering, Phys. Rev. X 8, 031014 (2018).
[17] R. C. Nelson and J. T. Miller, An introduction to X-ray absorption spectroscopy and its in situ application to organometallic compounds and homogeneous catalysts, Catal. Sci. Technol. 2, 461 (2012).

[18] M. Rioult, D. Stanescu, P. Le Fèvre, A. Barbier, and H. Magnan, Resonant photoemission spectroscopy investigation of $\mathrm{Fe}_{2} \mathrm{O}_{3}-\mathrm{TiO}_{2}$ heterojunctions for solar water splitting, Phys. Proc. 85, 4 (2016).

[19] D. W. Boukhvalov, M. Al-Saqer, E. Z. Kurmaev, A. Moewes, V. R. Galakhov, L. D. Finkelstein, S. Chiuzbăian, M. Neumann, V. V. Dobrovitski, M. I. Katsnelson, A. I. Lichtenstein, B. N Harmon, K. Endo, J. M. North, and N. S. Dalal, Electronic structure of a $\mathrm{Mn}_{12}$ molecular magnet: Theory and experiment, Phys. Rev. B 75, 014419 (2007).

[20] A. Saywell, A. J. Britton, N. Taleb, M. del Carmen GiménezLópez, N. R. Champness, P. H. Beton, and J. N. O'Shea, Single molecule magnets on a gold surface: In situ electrospray deposition, x-ray absorption and photoemission, Nanotechnology 22 , 075704 (2011).

[21] S. A. Wilson, T. Kroll, R. A. Decreau, R. K. Hocking, M Lundberg, B. Hedman, K. O. Hodgson, and E. I. Solomon, Iron L-edge x-ray absorption spectroscopy of oxy-picket fence porphyrin: Experimental insight into $\mathrm{Fe}-\mathrm{O}_{2}$ bonding, J. Am. Chem. Soc. 135, 1124 (2013).

[22] J. J. Yan, T. Kroll, M. L. Baker, S. A. Wilson, R. Decréau, M Lundberg, D. Sokaras, P. Glatzel, B. Hedman, K. O. Hodgson, and E. I. Solomon, Resonant inelastic X-ray scattering determination of the electronic structure of oxyhemoglobin and its model complex, Proc. Natl. Acad. Sci. (USA) 116, 2854 (2019).

[23] C. S. Ponseca, P. Chábera, J. Uhlig, P. Persson, and V. Sundström, Ultrafast electron dynamics in solar energy conversion, Chem. Rev. 117, 10940 (2017).

[24] E. Coronado, Molecular magnetism: From chemical design to spin control in molecules, materials and devices, Nat. Rev. Mater. 5, 87 (2020).

[25] V. Balzani, G. Bergamini, S. Campagna, and F. Puntoriero, Photochemistry and photophysics of coordination compounds: Overview and general concepts, Top. Curr. Chem. 280, 1 (2007).

[26] P. Glatzel, U. Bergmann, W. Gu, H. Wang, S. Stepanov, B. S. Mandimutsira, C. G. Riordan, C. P. Horwitz, T. Collins, and S. P. Cramer, Electronic structure of Ni complexes by $\mathrm{X}$ ray resonance Raman spectroscopy (resonant inelastic X-ray scattering), J. Am. Chem. Soc. 124, 9668 (2002).

[27] K. Kunnus, W. Zhang, M. G. Delcey, R. V. Pinjari, P. S. Miedema, S. Schreck, W. Quevedo, H. Schröder, A. Föhlisch, K. J. Gaffney, M. Lundberg, M. Odelius, and P. Wernet, Viewing the valence electronic Structure of ferric and ferrous hexacyanide in solution from the Fe and cyanide perspectives, J. Phys. Chem. B 120, 7182 (2016).

[28] R. H. Temperton, S. T. Skowron, K. Handrup, A. J. Gibson, A. Nicolaou, N. Jaouen, E. Besley, and J. N. O'Shea, Resonant inelastic X-ray scattering of a Ru photosensitizer: Insights from individual ligands to the electronic structure of the complete molecule, J. Chem. Phys. 151, 074701 (2019).

[29] S. S. N. Lalithambika, K. Atak, R. Seidel, A. Neubauer, T. Brandenburg, J. Xiao, B. Winter, and E. F. Aziz, Chemical bonding in aqueous hexacyano cobaltate from photonand electron-detection perspectives, Sci. Rep. 7, 40811 (2017). 
[30] R. H. Temperton, N. W. Rosemann, M. Guo, N. Johansson, L. A. Fredin, O. Prakash, K. Wärnmark, K. Handrup, J. Uhlig, J. Schnadt, and P. Persson, Site-selective orbital interactions in an ultrathin iron-carbene photosensitizer film, J. Phys. Chem. A 124, 1603 (2020).

[31] J. S. Griffith and L. E. Orgel, Ligand-field theory, Q. Rev. Chem. Soc. 11, 381 (1957).

[32] J. J. Sakurai, Advanced Quantum Mechanics (Addison-Wesley, Reading, MA, 1967).

[33] B. Winter, R. Weber, W. Widdra, M. Dittmar, M. Faubel, and I. V. Hertel, Full valence band photoemission from liquid water using EUV synchrotron radiation, J. Phys. Chem. A 108, 2625 (2004).

[34] See Supplemental Material at http://link.aps.org/supplemental/ 10.1103/PhysRevResearch.3.033030 for a valence-band spectrum showing the water $2 \mathrm{a}_{1}$ peak used for calibration, an example of the peak fitting procedure used and the two RPES maps showing the full binding energy range measured.

[35] R. Seidel, M. N. Pohl, H. Ali, B. Winter, and E. F. Aziz, Advances in liquid phase soft-x-ray photoemission spectroscopy: A new experimental setup at BESSY II, Rev. Sci. Instrum. 88, 073107 (2017).

[36] T. Kachel, The plane grating monochromator beamline U49-2 PGM-1 at BESSY II, J. Large-scale Res. Facilities 2, A72 (2016).

[37] R. K. Hocking, E. C. Wasinger, F. M. De Groot, K. O. Hodgson, B. Hedman, and E. I. Solomon, Fe L-edge XAS studies of $\mathrm{K}_{4}\left[\mathrm{Fe}(\mathrm{CN})_{6}\right]$ and $\mathrm{K}_{3}\left[\mathrm{Fe}(\mathrm{CN})_{6}\right]$ : A direct probe of back-bonding, J. Am. Chem. Soc. 128, 10442 (2006).

[38] N. Engel, S. I. Bokarev, E. Suljoti, R. Garcia-Diez, K. M. Lange, K. Atak, R. Golnak, A. Kothe, M. Dantz, O. Kühn et al., Chemical bonding in aqueous ferrocyanide: Experimental and theoretical X-ray spectroscopic study, J. Phys. Chem. B 118, 1555 (2014).

[39] R. V. Pinjari, M. G. Delcey, M. Guo, M. Odelius, and M. Lundberg, Restricted active space calculations of L-edge X-ray absorption spectra: From molecular orbitals to multiplet states, J. Chem. Phys. 141, 124116 (2014).

[40] R. Seidel, S. Thürmer, J. Moens, P. Geerlings, J. Blumberger, and B. Winter, Valence photoemission spectra of aqueous
$\mathrm{Fe}^{2+/ 3+}$ and $\left[\mathrm{Fe}(\mathrm{CN})_{6}\right]^{4-/ 3-}$ and their interpretation by DFT calculations, J. Phys. Chem. B 115, 11671 (2011).

[41] G. B. Armen, H. Aksela, T. Åberg, and S. Aksela, The resonant Auger effect, J. Phys. B 33, R49 (2000).

[42] M. J. Frisch, G. W. Trucks, H. B. Schlegel, G. E. Scuseria, M. A. Robb, J. R. Cheeseman, G. Scalmani, V. Barone, B. Mennucci, G. A. Petersson, H. Nakatsuji, M. Caricato, X. Li, H. P. Hratchian, A. F. Izmaylov, J. Bloino, G. Zheng, J. L. Sonnenberg, M. Hada, M. Ehara, K. Toyota, R. Fukuda, J. Hasegawa, M. Ishida, T. Nakajima, Y. Honda, O. Kitao, H. Nakai, T. Vreven, J. A. Montgomery, Jr., J. E. Peralta, F. Ogliaro, M. Bearpark, J. J. Heyd, E. Brothers, K. N. Kudin, V. N. Staroverov, T. Keith, R. Kobayashi, J. Normand, K. Raghavachari, A. Rendell, J. C. Burant, S. S. Iyengar, J. Tomasi, M. Cossi, N. Rega, J. M. Millam, M. Klene, J. E. Knox, J. B. Cross, V. Bakken, C. Adamo, J. Jaramillo, R. Gomperts, R. E. Stratmann, O. Yazyev, A. J. Austin, R. Cammi, C. Pomelli, J. W. Ochterski, R. L. Martin, K. Morokuma, V. G. Zakrzewski, G. A. Voth, P. Salvador, J. J. Dannenberg, S. Dapprich, A. D. Daniels, O. Farkas, J. B. Foresman, J. V. Ortiz, J. Cioslowski, and D. J. Fox, Gaussian 09, Revision E.01 (Gaussian, Inc., Wallingford, CT, 2013).

[43] M. Reiher, O. Salomon, and B. Artur Hess, Reparametrization of hybrid functionals based on energy differences of states of different multiplicity, Theor. Chem. Acc. 107, 48 (2001).

[44] F. Weigend and R. Ahlrichs, Balanced basis sets of split valence, triple zeta valence and quadruple zeta valence quality for $\mathrm{H}$ to Rn: Design and assessment of accuracy, Phys. Chem. Chem. Phys. 7, 3297 (2005).

[45] B. P. Pritchard, D. Altarawy, B. Didier, T. D. Gibson, and T. L. Windus, An open, up-to-date resource for the molecular sciences community, J. Chem. Inf. Model. 59, 4814 (2019).

[46] D. Leshchev, T. C. Harlang, L. A. Fredin, D. Khakhulin, Y. Liu, E. Biasin, M. G. Laursen, G. E. Newby, K. Haldrup, M. M. Nielsen, K. Wärnmark, V. Sundström, P. Persson, K. S. Kjær, and M. Wulff, Tracking the picosecond deactivation dynamics of a photoexcited iron carbene complex by time-resolved X-ray scattering, Chem. Sci. 9, 405 (2018).

[47] J. Tomasi, B. Mennucci, and R. Cammi, Quantum mechanical continuum solvation models, Chem. Rev. 105, 2999 (2005). 